\title{
Commentary: Learning by Doing Biomedical Research—Value Added for Medical Education?
}

\author{
Michael J. Friedlander ${ }^{1,2,3}$ (D) \\ Published online: 1 October 2019 \\ (C) International Association of Medical Science Educators 2019
}

Abraham Flexner in his 1910 report [1] urged the training of physicians to practice in a scientific manner and the engagement of medical faculty in research. It has been noted that the Flexner report emphasized learning by doing and solving problems vs. traditional rote memorization. However, with all of its impact over the years in medical education, there have been concerns expressed that the "hyper-rational world of German medicine" while facilitating excellence in science was not accompanied by equivalent excellence in clinical caring [2]. Thus, the pendulum continues to swing - science, compassion, science, compassion, and so on. It is fair to ask whether this is a true dichotomy where a choice must be made to emphasize one at the expense of the other or of there can be consilience. After all, who among we patients doesn't want their health care provider to be armed with the best science that medicine has to offer for our care and also to be a compassionate person whose decisions are guided by principles that are both scientifically informed but also driven by true caring for what may be best for us? This challenge permeates the conference rooms of medical education offices across the nation and too often results in decisions where one aspect of healthcare education is pitted against the other.

Over the last nine years, I have had the privilege of working at one of the nation's newer medical schools - the Virginian Tech Carilion School of Medicine in Roanoke, Virginia, where these questions (and many others) could be looked at freshly and new approaches tried. While I was originally hired to lead the growth of the biomedical research enterprise as the executive director of a brand new research institute organized

Michael J. Friedlander

friedlan@vtc.vt.edu

1 Health Sciences and Technology, Virginia Tech, Roanoke, VA, USA

2 Fralin Biomedical Research Institute at Virginia Tech Carilion, Roanoke, VA, USA

3 Virginia Tech Department of Biological Sciences and Virginia Tech Carilion School of Medicine Department of Psychiatry and Behavioral Medicine, Roanoke, VA, USA in parallel with the new medical school and later was also appointed to lead larger scale health sciences initiatives across the Virginia Tech campuses as the university's vice president for health sciences and technology, I quickly also became immersed in the development and delivery of one aspect in particular of the nascent medical school's educational enterprise - the research value domain. I have had this opportunity through serving in yet another leadership role - that of senior dean for research at the medical school, upon the generous invitation of the medical school's founding dean, Dr. Cynda Johnson. It has been that position that has allowed me to be part of the discussions raised above about Flexner, the role of science and compassion in the delivery of twentyfirst century health care and how to build and deliver a program that nurtures tomorrow's thought leaders in medicine who are comfortable with and readily use science and compassion in a seamless fashion. This journey has been challenging and fulfilling for many of us here at the VTCSOM and has been a process that can offer some perspectives worth sharing with colleagues in other medical schools. In this commentary, I share a little bit about the program we have developed, how it seems to be working and how this may be useful to others.

As context for this commentary, I share a bit about my road along this path. In 2007, I had the honor of serving as the Chair of the Council of Academic Societies (CAS) of the Association of American Medical Colleges (AAMC) allowing me to participate in selecting the theme for the CAS spring meeting where we asked what value the research environment adds to the undergraduate medical education experience. While there were many informative presentations and rich discussions, I think it is safe to say that the consensus was that most meeting attendees felt there was considerable added value but proving it with data from appropriately controlled studies was not done easily. Fast forward to 2007-2009, when I had the privilege to serve on the AAMC-Howard Hughes Medical Institute (HHMI) task force on the Scientific Foundations of Future Physicians (SFFP) that explored the role of science in twenty-first century medical education. A 
very accomplished group of medical educators, physicians, scientists, and academic medicine and science leaders worked together for several years and submitted a report that called for increasing the emphasis for medical (and graduate) students' understanding of how data are obtained and weighing competing scientific claims; the practice of evidence-based medicine; earning and keeping the trust of the public/patients; being competent and appropriately confident to discuss/explain the scientific rationale of a healthy lifestyle, disease progression, diagnosis, and treatment to patients and the general public; keeping abreast of the relevant medical and scientific literature; understanding and utilizing genomics, bioinformatics, behavioral analyses, and critical reasoning skills to become life-long learners; being able to deal with the unexpected and having the skills to discern "pitch" from scientifically grounded information from any sources that might be more motivated by selling us their product than what is best for the patient or public. It was agreed that there could be many approaches to accomplish these goals through different curricula but that one possible component could be engaging medical students in the scientific discovery process throughout their undergraduate medical education years.

Thus, I was excited to have the opportunity in 2010 (the year after the SFFP report was published) to join the new enterprise at Virginia Tech Carilion that included my primary role to develop and direct the new biomedical research institute but also to serve as the senior dean for research at VTCSOM, working in close collaboration with the new VTC School of Medicine's founding dean, Dr. Cynda Johnson; the senior dean for academic affairs, Dr. Rick Vari; the assistant dean for research, Dr. Leslie LaConte; and many others to work together to put in place aspects of the SFFP, with an entire value domain centered on research. VTCSOM has the stated goal to educate physician thought leaders through inquiry, research, and discovery that incorporates four value domains throughout the four-year curriculum - basic science, clinical science, inter-professionalism, and research. The research domain that is woven throughout all four years incorporates a research mentor, an advisory committee, multiple oral, and written presentations on the student's hypothesis-driven original research project and culminates with a poster or platform presentation at a regional/national/international meeting, a written publication-quality manuscript (that may be published), and a formal presentation. Through this process, coupled with an integrated formal research curriculum that covers research methods, biostatistics, epidemiology, regulatory information, and a method in logic - visiting medical scholars program throughout the first year - we aim to graduate "scientist physicians."

Unlike traditional $\mathrm{MD} / \mathrm{PhD}$ program that produces physician scientists, our program is directed at the student who is primarily interested in becoming a practitioner of allopathic medicine, but who values the scientific approach for all aspects of the practice of medicine, including the humanistic and communications-based aspects of medicine. We define a scientist physician as one who: routinely utilizes and applies scientific reasoning in the practice of medicine; is an effective communicator of scientific rationale for decisions to patients; is motivated and capable of keeping abreast of scientific advances in medicine; knows where to go and who to go to for additional information on the scientific validity of claims of improved diagnostics and therapeutics; is an effective advocate for her patients for scientifically validated utilization of pharmaceuticals, devices, and procedures; is a critical and capable skeptic on behalf of his patients and able to discern "pitch" from scientifically valid claims of effectiveness; is willing and able to advance medicine by engaging in medical research; and bridges the gap between clinic and bench by communicating with scientists.

We have graduated six classes through 2019 where all graduates have completed their research projects and presented their work at a meeting. About $40 \%$ of the students have published either as first author, co-first-author, or as an embedded author. The students have $100 \%$ success in passing board exams (with excellent overall scores) and 100\% residency match rates. With only the first couple classes having completed residencies so far, it is still too early to determine how successful the program has been in having our graduates becoming practicing scientist physicians. However, we have learned several things during this period. Medical students are capable of participating in meaningful research integrated into a four-year curriculum; meaningful research does not negatively impact students' performance on board exams, acquisition and retention of clinical skills and it helps with residency placement; team work and inter-professionalism extend to the research domain; discovery, including failure and personal Eurekas can reinforce the other aspects of the curriculum; the type of research (basic, translational, clinical, community based) is not as important as the quality of the experience; most students enthusiastically embrace discovery learning and sharing what they've discovered; and most students consider their research experiences to have contributed to the quality of their medical education. Throughout the first nine years, we have seen students complete multiple very sophisticated research projects from the basic wet lab to the clinic to community outreach. While many projects began searching for a focused hypothesis with concern about whether the project may be completed in the allotted four years, whether the power of the study design would be sufficient and whether the goals were realistic, the first classes paved the way by owning their work, buying into the value proposition of the added value of their doing (vs. only reading about) biomedical/ health research and often mentoring their mentors as much as vice-versa as to strategy and implementation design to get the project on track and completed. While none of us knew exactly how things would turn out, I think it is safe to say that our expectations have at least been met and more likely exceeded. 
Whether this program will work as well at other institutions will of course depends on many factors including culture, faculty, students, and resources.

Time will tell if our approach succeeds in accomplishing our goals. In these times of multiple pressures on curricula, demands for decreasing the time in medical school to address the student debt issue and the physician shortage and in an age of increasing utilization of digital analytics, algorithms for the diagnosis and development of treatment plans including through artificial intelligence, one can reasonably argue that the development of scientist physician thought leaders is perhaps too expensive and not absolutely necessary. Certainly, there will be surprises still to emerge about how health care is delivered in the USA including the organization and funding of healthcare, the continuing development of technology, and possibly the emergence of health issues that are not currently mainstream as might emerge from climate change, emerging infections, massive social-political unrest and disruption and even the need for maintaining human life beyond the confines of the earth. It is likely that the kind of scientist physicians we are working to educate and train at VTCSOM who are compassionate yet scientifically grounded and un- afraid to meet these challenges will be an important contribution to the responses we mount to these challenges.

\section{Compliance with Ethical Standards}

Conflict of Interest The author declares that there is no conflict of interest.

Ethical approval NA.

Informed consent NA.

\section{References}

1. Flexner, A., Medical education in the United States and Canada: a report to the Carnegie Foundation for the advancement of teaching, 1910.

2. Duffy TP. The Flexner report -100 years later, Yale. J Biol Med. 2011;84(3):269-76.

Publisher's Note Springer Nature remains neutral with regard to jurisdictional claims in published maps and institutional affiliations. 